\title{
Protée
}

\section{Peirce et la clinique}

\section{Michel Balat}

Volume 30, numéro 3, hiver 2002

Autour de Peirce : poésie et clinique

URI : https://id.erudit.org/iderudit/006864ar

DOI : https://doi.org/10.7202/006864ar

Aller au sommaire du numéro

Éditeur(s)

Département des arts et lettres - Université du Québec à Chicoutimi

ISSN

0300-3523 (imprimé)

1708-2307 (numérique)

Découvrir la revue

\section{Citer cet article}

Balat, M. (2002). Peirce et la clinique. Protée, 30(3), 9-24.

https://doi.org/10.7202/006864ar

\section{Résumé de l'article}

Cet article présente les principaux concepts dont nous avons pu montrer jusqu'ici l'efficience dans la théorie psychanalytique. Tous sont tirés de l'œuvre de Peirce, sa phanéroscopie et sa sémiotique. Leur justification peut être trouvée dans nos différents ouvrages. Nous tentons à travers eux une articulation propre au champ de la clinique. Nous proposons une nouvelle idée, celle de forme-émergente ou forme-rythme afin de rendre compte de l'impossibilité de considérer la forme comme un objet défini. Il nous semble que cela peut constituer une avancée dans la prise en compte de la logique du vague de Peirce. 


\section{PEIRCE ET LA CLINIQUE}

MiCHEL BALAT

\section{INTRODUCTION}

Depuis l'origine de la clinique, c'est-à-dire depuis Hippocrate, la question du signe est centrale. Elle l'a été tout d'abord pour la question du pronostic: comment, à partir des rapports divers que le médecin peut établir avec le malade - et dans un premier temps, essentiellement dans les positions par rapport à son lit, d'où le terme de "clinique», à savoir du plus loin, le pas de la porte, au plus près, le chevet-, dire ce qu'il va advenir de lui: la mort? la guérison? On pourrait dire, en employant ce terme de la philosophie des scolastiques que Peirce a repris avec bonheur, qu'en ce premier temps s'élaborait une «grammaire spéculative», c'est-àdire la définition des conditions pour qu'un signe soit un signe. Je cite souvent un petit événement qui me paraît très intéressant: nous avons formé depuis de nombreuses années un petit groupe de médecins pour saisir l'incidence de la relation entre le praticien et son client en ce qui concerne le soin. Lors d'une de ces séances, était discuté un court texte d'Hippocrate ${ }^{1}$, il s'agit du "cas Philiscos», dans Épidémies $I^{2}$. Mes amis discutaient de ce cas et hésitaient sur le diagnostic jusqu'au moment où l'un d'entre eux fit remarquer que Philiscos habitait près du rempart et émit le diagnostic de "fièvre bilieuse hémoglobinurique», diagnostic habituellement posé par la cohorte des médecins après Hippocrate, cette fièvre étant liée aux suites du paludisme contracté en climat très humide.

Il serait possible, à partir de là, de faire l'histoire de cette grammaire spéculative à travers les progrès et le régrès de la médecine. Mais ce ne sera pas notre chemin.

Nous voulons simplement indiquer que la question du signe est indissolublement liée à la clinique, au point où nous pouvons même penser que c'est dans ce champ qu'ont été maintenues concrètement et sérieusement les interrogations sémiotiques, plus qu'en philosophie. Au cours des siècles, la partie de la médecine consacrée à la discrimination et à l'étude des causes des signes s'appelait la «séméiotique».

Peirce lui-même ne s'est jamais (ou très peu) référé à l'histoire de ce champ dans l'élaboration de sa sémiotique. Nous nous appuyons donc depuis toujours sur sa sémiotique, mais pas sur les rapports qu'il aurait déjà pu élaborer par lui-même. Par ailleurs le monde médical n'a pas beaucoup évolué dans l'étude d'une 
sémiotique générale, et ce n'est pas l'utilisation massive, dans les articles médicaux, d'inductions ou de pseudo-inductions qui serait propre à modifier notre point de vue. Seule, peut-être, la réflexion psychiatrique échappe à cette constatation, mais sans doute devons-nous savoir que son champ a été considérablement transformé par les travaux de Freud, chez qui il y a une profonde étude épistémologique de la question du signe. Aussi nos propres travaux ont-ils commencé par l'étude des rapports de la sémiotique de Peirce et de la psychanalyse freudienne ${ }^{3}$. Depuis, le champ s'est élargi aux questions posées par l'éveil du coma, puis par l'autisme, et aux relations dans les établissements de soin. C'est dire que la problématique de la clinique et son abord "poiétique» est bien plus large que ce que nous pouvons proposer ici: nous nous bornerons à une approche de cette question par le biais de la psychanalyse, des questions de l'éveil du coma et de l'autisme, de la psychiatrie et de la psychothérapie institutionnelle (s'il est possible de distinguer les deux derniers).

Je me limiterai dans les pages qui suivent à la présentation de quelques-uns des concepts ou de quelques-unes des notions sémiotiques qui nous sont d'un grand secours dans la clinique concrète, quotidienne, qui est la nôtre 4 .

\section{LA PHÉNOMÉNOlogie de PEIRCE}

Sous le nom de "phanéroscopie», Peirce aborde les problèmes phénoménologiques à l'aide d'un opérateur formel puissant, les catégories de Priméité, Secondéité et Tiercéité, permettant d'extraire de tout phénomène ou "phanéron" (ce qui est présent à l'esprit) différents éléments dont le contenu et la structure d'ensemble sont clairement révélés. $\mathrm{La}$ méthode, simple à exposer mais complexe à mettre en œuvre, est dialectique. Rappelons très brièvement ce que sont ces catégories. La Priméité est la catégorie de ce qui est tel qu'il est, indépendamment de quoi que ce soit d'autre: nous pourrions dire que c'est l'être, vieux concept de la philosophie, mais à condition de le considérer pour l'essentiel comme le «possible». La
Secondéité est l'être de ce qui est par autre chose et recouvre pratiquement le concept d'existence. Enfin la Tiercéité est l'être de ce qui est en mettant en relation un premier et un second, en somme le signe. Ces catégories sont ordonnées par la préscission, c'est-à-dire le fait que la secondéité implique la priméité, mais pas le contraire, et que la tiercéité implique la secondéité et la priméité, mais pas le contraire. La méthode est la suivante: le sujet de l'enquête étant posé, on étudiera sa priméité, et celle-ci se révélera être la priméité (ou l'être) de la priméité, la priméité de la secondéité ou la priméité de la tiercéité. Puis nous examinerons la secondéité et la tiercéité. Mais de fait, chaque fois, ce que nous aurons produit sera toujours l'être de la chose, sa priméité, dès lors nous pourrons continuer l'analyse aussi loin que nous le pourrons en posant que, par exemple, la priméité de la secondéité que nous avons produite n'est autre que la priméité de sa priméité. Ce retour incessant sur le phénomène nous permet d'en produire l'analyse ainsi que la structure. Bien entendu tout phanéron «contient» de la tiercéité (sans quoi nous ne pourrions même le concevoir), mais cette tiercéité n'est pas nécessairement sa priméité, d'une part, et, d'autre part, si elle l'était, il aurait néanmoins une "part» de secondéité et une de priméité.

En conséquence, nous pouvons envisager des "catégories dégénérées", au sens où un sujet clairement second n'aurait pas comme priméité la secondéité: nous dirions à ce moment-là que sa secondéité est dégénérée. De même, un troisième pourrait avoir comme priméité la secondéité, et on dirait alors que sa tiercéité est dégénérée une fois, ou comme priméité, auquel cas sa tiercéité serait dite dégénérée deux fois. De multiples travaux ont été consacrés à ces notions ${ }^{5}$, nous y renvoyons pour une étude plus complète.

Dans la pratique la plus concrète, où ces catégories ont un emploi, nous les retrouvons sous les espèces de la forme ou de la qualité pour la priméité, de l'existence ou de la force brute pour la secondéité, du signe ou de la continuité pour la tiercéité. Il nous faudrait examiner chacune de ces idées fondamentales 
liées aux catégories pour montrer en quoi elles reçoivent un sens différent d'être prises dans l'opérateur dont nous avons parlé. Nous nous contenterons ici de considérer la priméité et ses deux idées typiques que sont la forme et la qualité. Certes ce sont des "priméités", autrement dit des possibles. Mais peut-être n'a-t-on pas suffisamment perçu (et je parle ici au moins pour moi!) que le terme possible est souvent pris dans un sens restrictif. En effet, il est souvent pensé par rapport à l'impossible, soit dans une forme de secondéité. Par exemple «il est possible qu'il pleuve» fait référence au fait qu'il pleuve ou qu'il ne pleuve pas, donc à un certain dyadisme latent. La possiblité dont il est question ici, à ce niveau fondamental de la priméité, est une sorte de possibilité de la possibilité, un niveau où aucune forme et aucune qualité, pour aussi typique qu'elle soit, n'est totalement définie. Nous proposerions bien, tout en sachant qu'il n'y a aucun terme correct pour formuler cela, l'idée de «forme émergente» ou de "qualité émergente», à condition d'enlever à l'émergence toute idée d'espace et de temps, comme les physiciens conçoivent la «vitesse immédiate» ou la «dérivée»: comme nous le verrons en conclusion, nous pourrions aussi bien employer le terme de «rythme», propre à rendre compte de l'idée de processus latent. Une forme, une qualité émergente, en tant qu'elle est une possibilité de forme ou de qualité, n'a pas d'identité propre. Ce n'est qu'en recevant de ce dans quoi elle s'incorpore de la secondéité ou de la tiercéité qu'elle aura une certaine identité ou une stabilité structurelle. Mais en ellemême, dans sa pure priméité, elle est émergence de formes ou de qualités. Il y a ici tout un champ déjà parcouru par toute une série de penseurs de la phénoménologie à propos de la Gestaltung.

Il nous semble que ceci fournit la racine même des réflexions de Peirce sur la logique du vague.

Comment pourrait-il y avoir quelque logique du vague s'il n'y avait précisément des qualités émergentes, sans identité propre, pour tout dire, largement indéfinies. Car l'indéfinition n'est pas simplement dans le signe présentant l'objet, elle se trouve plus fondamentalement dans la forme même ou dans la qualité qui y prend corps. L'identité d'une qualité est seconde par rapport à la qualité émergente; et nous voyons clairement ici comment la qualité conçue fait le lien entre la qualité émergente et celle qui a une identité. Plus prosaïquement, par exemple, le rouge (3e) met en relation la rougéité (1 $\mathrm{re}$ ) avec le rouge (2e) incorporé dans cet objet.

\section{LE TONAL}

Nous avons, depuis plus de 10 ans, proposé une notion, celle de «tonal», pour rendre compte de l'ensemble des qualités ou des formes qui constituent le socle commun que partagent des hommes dans leurs rapports (relationships). Cette notion nous était dictée par le travail réalisé en collectif auprès des traumatisés crâniens et elle s'est avérée très pratique pour qualifier le socle institutionnel des établissements de soin, mais aussi la qualité de présence que l'on peut percevoir dans le travail psychanalytique. Dans la mesure où les rapports humains sont fondamentalement des rapports de signes, c'est au "Ton», dimension poétique ou poïétique de la sémiotique peircienne, que nous avions fait appel. Rappelons ici que le Ton (tone) est cette modalité du "représentement" 6 qui, d'une qualité, fait un signe, par une sorte d'accrétion de tiercéité. Du fait même de cette accrétion, il y a une sorte de mise en continuité des signes impliqués qui donne ce caractère de tissu des signes où, précisément, les qualités émergentes sont largement à l'œuvre. Nous avons trichotomisé ces tons en produisant une distinction entre l'Homoton, soit la priméité du ton, le Synton, sa secondéité, et le Diaton, sa tiercéité. Cette opération était légitimée par l'accrétion: il fallait bien qu'il y eût quelque chose comme un «diaton" pour que le «ton» fût possible (respect de la hiérarchie des catégories). L'ensemble de cette opération nous permettait de rendre compte du fait que la parole partagée peut avoir une influence sur le tonal, sous certaines conditions liées au caractère, à la modalité de ce qu'est le ton. Par exemple, la voix dans sa dimension de prosodie, sa texture, le moment où elle advient, mais aussi la potentialité signifiante 
qu'elle possède sont des éléments fondamentaux de transformation du tonal. Nous avons à plusieurs reprises présenté les conditions concrètes où se jouaient ces transformations tonales et les effets auxquels cela donnait lieu. Mais sans doute faut-il situer plus précisément l'articulation du «tonal» par rapport aux grandes fonctions de la parole.

INTRODUCTION À L'INSCRIPTION, AU MUSEMENT ET À L'INTERPRÉTATION

Pour tenter de définir les fonctions qui sont concernées par le fait d'être un être de langage, celles qui sont impliquées dans l'exercice du langage, il faudrait pour cela se tourner, semble-t-il, vers les linguistes. Le plus clair sur cette question est, sans doute, Benveniste, même si sa démarche est obstinément dyadique: pour lui, tout est divisible par deux. Il est entre autres l'auteur de ce livre génial, Le Vocabulaire des institutions indo-européennes, un livre de base, où il propose une théorie de la racine des mots d'une langue originelle. Il a donc créé un vocabulaire d'une utilité phénoménale.

Benveniste a également créé les grandes fonctions attachées à la parole, soit la fonction de l'énoncé et la fonction de l'énonciation. De manière un peu rapide, nous pourrions dire que l'énoncé, c'est ce qui est écrit, et l'énonciation, l'acte d'assomption, de production de la chose écrite. Un écrit a une histoire, un moment où il prend corps et ce moment, c'est l'énonciation. C'est utile dans certaines distinctions, comme celle qui est opérée entre le sujet de l'énoncé et le sujet de l'énonciation: quand je dis une phrase, le «je» est sans aucun doute le sujet de l'énoncé, mais pas le sujet de l'énonciation, qui est la production de la phrase et les conditions dans lesquelles je suis amené à produire cette phrase - un autre registre. Benveniste a été repris de manière très fine par Lacan, qui en a fait un outil fondamental de la clinique psychanalytique, élaborant des conceptions comme celle de la division du sujet.

Étant en désaccord avec cette démarche de Benveniste, il m'a semblé, en m'appuyant sur C.S. Peirce, que l'on pouvait reprendre cette question des grandes fonctions, et en observer trois. Ce n'est pas parce qu'il y en a trois, ce n'est pas trois contre deux, mais trois qui sont articulées et dont aucune n'est réductible à l'autre. En énonçant les trois grandes fonctions, nous verrons par la suite comment nous pouvons les articuler.

La première fonction de base est celle que j'appelle la fonction scribe - Peirce en parle dans quelques passages de son œuvre. Une deuxième fonction fondamentale est la fonction museur et la troisième est la fonction interprète.

\section{LA FONCTION SCRIBE}

La fonction scribe, c'est la possibilité même que quelque chose puisse connaître une certaine matérialisation: quand je parle, j'écris, cela nécessite quelque concrétude. Si je parle mais qu'aucun son ne sorte de ma bouche, rien ne va s'inscrire: de telle manière qu'il y a sans doute un moment d'origine dans lequel il est indispensable de définir quelque chose qui ait une certaine hylé. Mais celle-ci ne suffit pas. Supposons qu'au lieu de parole, soit produit un brouhaha. Il y aura du son, c'est le sens du mot brouhaha, qui est un son indistinct, incompréhensible. Au sein d'une foule, il y a un brouhaha, ce sont peut-être des paroles, c'est sonore, assurément - je ne fais pas référence à la musique qui est organisée -, le brouhaha est une espèce de chaos sonore duquel rien ne sort. Nous voyons les deux pôles, la matérialité, mais il ne peut pas y avoir que la matérialité - si j'écris avec des pattes de mouches, de manière illisible, le lecteur sera encore dans l'expectative, ce qui montre que cette fonction scribe nécessite une certaine concrétude -, il doit y avoir en sus une fonction que nous allons qualifier de fonction d'inscription.

L'inscription, c'est autre chose que la matérialité de l'écriture, mais c'est quelque chose qui fait appel à une matérialité. J'inscris, mais au sens où, par exemple, l'enfant, par son corps, inscrit quelque chose. Nous ne faisons pas ici référence uniquement à la langue, ni à l'écrit tels qu'ils sont organisés: cette hylé peut être celle du corps, et sans doute même pouvons-nous 
penser que de toute façon, et à un moment originaire, il faut bien que cette fonction scribe fût d'abord et avant tout une fonction attachée au corps. Je précise que cette inscription est une fonction qui s'inscrit sur le leib, sur le corps vivant, dans le sens que lui donne Jean Oury - nous pourrions dire le corps sémiotique-, en le distinguant du körper. Cette fonction

d'inscription, comme fonction de base sans doute, de manière originaire, nécessite le corps. Le nourrisson doit pouvoir inscrire, c'est-à-dire découper son corps pour pouvoir l'utiliser «comme un langage». Les premiers rapports de la mère et de l'enfant semblent indiquer cela, comme lorsque la maman voit gigoter son petit et qu'elle dit: «il est content». Gigoter, c'est du leib, c'est un morceau de leib, il faut mettre les pieds et les jambes d'une certaine façon. Au troisième mois, le premier organisateur de Spitz ${ }^{7}$, le sourire ( il est content») ou bien le premier pleur: («il a faim»): c'est bien toujours en référence au corps que la mère vient introduire de la parole.

On peut dire que la fonction scribe est une fonction matérielle d'inscription, et cette fonction est une fonction originaire puisque c'est elle qui, dans un premier temps, se présente à l'enfant. Nous verrons plus loin la place de la mère, mais, pour le moment, disons que certains éléments, le tonus, le système musculaire, les voies de conductions nerveuses, l'organisation cérébrale, etc. - on pourrait parler de toutes ces choses-là, et de manière très utile, en faisant référence à ce que Freud appelait le "Real Ich", le moiréel - assurent la matérialité. Par exemple, si nous écrivons «a» à l'envers, nous pouvons le lire, mais si nous pratiquons certaines déformations, nous ne pourrons plus lire le «a». C'est une bonne analogie pour faire comprendre ce qui se passe à propos du corps; ce sont des «a» qu'il faut former, il faut trouver tout un ensemble de mouvements: ce n'est pas le körper, c'est le leib, c'est le corps en émergence de signes, un corps vivant, porteur de signes. Beaucoup plus tard, dans certains phénomènes somatiques, nous trouverons des formes d'archaïsmes, une forme de langage qui ne peut plus être exprimé dans l'universel de la langue, où seul le corps peut venir dire quelque chose - mais on ne sait quoi, puisque nous avons perdu la clé de ce langage. Nous pouvons trouver encore cela, mais d'une autre façon, dans les phénomènes hystériques, où il y a les «ha, j'ai mal à la tête, je ne vais pas pouvoir aller à...», ou bien «j'ai de la tachycardie» ou bien «j'ai de la spasmophilie», toutes ces choses qui sont la vie élémentaire végétative d'une ou d'un hystérique et qui sont sans doute des restes d'une autre nature que les somatisations. Mais ce sont des formes particulières d'appel à un langage corporel, et, contrairement aux somatisations, la clé de ce langage n'est pas dans le langage lui-même, comme cela se produit dans la somatisation, mais elle est dans l'appareil symbolique de la langue, ce qui évidemment ne relève pas du même type d'approche ou d'analyse.

Quand nous parlons, nous nous entendons. Mais comment nous entendons-nous? Ce n'est pas la voix que nous pourrions enregistrer sur un magnétophone. Nous nous entendons par la voie osseuse, par le squelette; c'est le squelette qui vibre. Nous avons quelque chose dans le larynx qui vibre, ce sont les cordes vocales. Elles font sifflet (d'ailleurs lorsqu'on veut couper la parole à quelqu'un, nous disons qu'on lui coupe le sifflet) et ce petit sifflet, ce muscle, fait vibrer les os du crâne, et c'est par eux que nous entendons notre propre parole. Le son se déplace plus vite dans un milieu plus solide, la vitesse dépend de la densité. Le son se déplace plus vite dans tout le système des os du crâne, et c'est lui qui arrive le premier à la perception de l'oreille interne. Le deuxième type de son est celui qui arrive par l'extérieur. Imaginons que quelques millièmes de secondes après avoir parlé, nous entendions en écho notre propre parole, ce serait insupportable. Il y a un système d'inhibition particulier qui fait que nous sommes accoutumés à ne pas entendre notre propre parole telle qu'elle passe par la voie aérienne: nous ne nous entendons pas comme nous entendons les autres. Les autres ne nous font pas vibrer les os par leur parole, ils font vibrer le tympan directement par la colonne d'air modulé qui provoque l'onde sonore, alors que nous, nous percevons par l'intermédiaire de la transmission osseuse. 
Cette inhibition fondamentale du circuit de notre parole "externe" évite le phénomène de réplication ou d'écho (peut-être est-ce même à l'origine du mythe d'Écho avec Narcisse). Imaginons la situation suivante, dans laquelle cette inhibition ne fonctionnerait pas et où nous entendrions toujours notre voix osseuse, mais aussi notre voix "aérienne", comme, par analogie, celui qui souffre d'une légère diplopie: il peut très bien s'en accommoder parce que c'est comme ça qu'il a toujours vu. On pourrait peutêtre même faire l'hypothèse que, chez l'enfant bègue, la parole correspond à ces deux niveaux d'écoute par manque d'inhibition. Cette hypothèse, je la présente non pas pour elle-même, mais pour explorer le fait qu'on ne s'entende pas comme nous entendons les autres, ce qui a assurément un sens. La parole que nous entendons est celle qui fait vibrer le squelette.

Voici une petite expérience dont je me serais passé très largement. Il y a quelque temps, en gare de Bordeaux, je me préparais à sortir du train avec mon sac un peu lourd. Alors que je descendais l'escalier, le sac s'est un peu balancé et m'a entraîné: je me suis senti tiré par ce sac, et je suis tombé lamentablement. C'était certes lamentable, mais il m'est arrivé quelque chose de magnifique: j'ai entendu sonner mon squelette, il a fait un son de caisse claire.

Dans l'acte de parole, c'est le corps entier qui est mobilisé, il est parcouru par les ondes sonores. Ce son de caisse claire montre bien que l'ensemble du squelette est un résonateur. Le squelette vibrant est quelque chose qui habite le corps tout entier et, quand nous parlons, la parole que nous entendons, émise de nous, est une parole totalement corporelle, c'est une vibration du corps tout entier. On pourrait, à partir de là, reprendre la grande question freudienne de la pulsion de mort.

Cette chose ne nous arrive pas quand nous entendons quelqu'un d'autre parler, il n'y a pas de vibration du corps. La seule chose qui vibre ce sont tous les osselets de l'oreille, tout ce monde qui fait son petit travail, l'usine, le marteau, l'enclume et tutti quanti. Les appareils de la perception sont des filtres tout à fait extraordinaires du monde extérieur.
L'oreille est destinée officiellement à pouvoir entendre le choc d'un atome sur le tympan, mais officiellement il n'y a pas de limite inférieure à l'audition (je ne parle pas de la fréquence, mais de l'intensité).

Il n'est pas sûr que tout un ensemble d'expériences menées généralement par les adolescents dans les boîtes de nuit n'aient pas quelque chose à voir avec ce que je viens de dire: cette vibration du corps ressentie près des enceintes acoustiques, c'est quelque chose qui n'est pas sans rappeler précisément sa propre parole, un peu comme si l'on émettait sa propre musique. Ce sont, dans ces conditions, des émissions de sa propre musique par le corps. C'est une manière de l'adopter totalement. Nous prenons entièrement la musique comme si elle émanait de notre propre corps, en raison de toute cette conduction osseuse.

Il en est de même au cinéma, où il y a une vibration corporelle: le son est si puissant qu'il fait vibrer le corps, comme si chacun était habité par les dialogues du film. Nous pourrions dire en somme que, lorsque je parle, ce n'est pas l'émission vocale que j'entends, comme pourrait la produire l'autre vis-à-vis de moi, puisque je ne l'entends pas par cette voie-là, mais par la vibration produite à partir de la caisse de résonance qu'est mon corps. Qu'est cet icelui qui parle par le corps?

Faisons une hypothèse: c'est comme si c'était l'Autre qui me parlait. Quand je parle, c'est l'autre, le corps, le leib mis en émoi par les paroles vibrantes, qui parle, c'est l'Autre. On peut dire qu'il y a quelque chose de l'Autre en moi qui parle. L'Autre, celui qui est vraiment le résonateur de base, qui est capable d'inscrire mes propres paroles, ce lieu-là, c'est ce leib, qui est l'Autre résonant. L'Autre, c'est moi en tant que je résonne. Comme l'indiquait Lacan, le grand Autre, l'Autre, c'est le corps - le leib ajouterait sans doute Jean Oury. L'Autre est indissolublement lié à la fonction scribe.

\section{LA FONCTION MUSEUR}

La deuxième grande fonction est la fonction museur. Le musement, de même que le scribe, est une idée de Peirce, mais aussi de Chrétien de Troyes, dans 
Perceval ou le Conte du Graal ${ }^{8}$. Dans ce conte, il y a quelque chose de magnifique. Nous croyons que le Graal est l'objet inatteignable, l'inaccessible étoile de Jacques Brel, et pourtant ce n'est pas vrai! Perceval a déjà été en contact avec le Graal. L'histoire se passe dans le château du roi Pêcheur. Perceval arrive et voit passer devant lui des gens qui font des choses bizarres, l'un avec une lance au bout de laquelle goutte du sang, un autre transporte un objet, le Graal. Il était certes intrigué par la scène, sans doute des questions lui venaient, mais il n'en a posé aucune (un vrai petit obsessionnel). C'est à partir de là que commence la quête du Graal, c'est-à-dire de cet objet qui est un objet perdu, un vrai objet. Par la suite, Perceval dort avec Blanchefleur, avec l'épée dans le mitan du lit (tout cela est très initiatique), puis part à la recherche du roi Arthur. C'est là qu'arrive le musement, en chemin. Il y a une oie blessée qui laisse tomber trois gouttes de sang sur la neige. Perceval, devant ces trois gouttes de sang, est non pas médusé ou stupéfait, il est en arrêt sur son cheval, appuyé sur sa lance, devant ces gouttes de sang, et là, il muse. Le verbe est de Chrétien de Troyes, en vieux français. Évidemment, ce mot n'est pas traduit par muser, puisque ce verbe n'existe plus en français dans ce sens-là, mais dorénavant il faudrait qu'il existe, c'est indispensable. Il muse sur ces gouttes de sang. Au loin, un chevalier du roi Arthur passe, le voit et retourne chez le roi pour annoncer la nouvelle. C'est un nommé Kex qui va chercher Perceval: le maladroit se précipite sur Perceval et lui intime de venir. Mais Perceval musait sur les gouttes de sang - c'est une activité à temps plein, on ne peut pas faire autre chose - et, tout en musant, il se bat avec Kex et le blesse. Ce dernier va se plaidre au roi Arthur et Gauvain propose alors d'aller chercher Perceval. Gauvain saisit qu'il se passe quelque chose de très important pour Perceval: ce n'est que lorsque les gouttes de sang ont fini par disparaître de la neige que Perceval peut être approché. Gauvain s'approche «en oblique», pour respecter ce musement, et amène Perceval chez le roi Arthur. Astucieux. Sur le plan clinique, c'est très important: la manière dont on approche quelqu'un, ce ne peut être de plein fouet - peut-être est-il en train de muser.

Je ne suis pas le seul à avoir cherché à inventer une étymologie au musement, mais il y en a une qui me paraît sérieuse: c'est le même mot que muet (pas le verbe muer de la mue), être muet, qui vient de museau, moue. En latin, être muet se disait faire $m u$; le musement est lié à la mutité, c'est une fonction du silence.

Qu'est-ce que cette fonction? Nous voyons que, dans son extrême, c'est une fonction dans laquelle le cours des pensées n'est pas dévié, il est lié à la perception, créé ou au moins soutenu par elle, et c'est un état continu, de base, quelque chose qui est en développement. Le musement, si on essaie de le saisir sur le plan phénoménologique, c'est ce qui arrive quand nous sommes dans le même état que Perceval: arrêté, un peu hors du monde. Il y a une perception sur laquelle le musement se soutient - toujours la question de la matérialité-, mais la perception, c'est l'occasion du musement. Cela se produit souvent: tout à coup nous restons en arrêt devant quelque chose, nous ne savons pas quoi, nous ne savons même pas que nous regardons, mais nous sommes pris dans nos pensées; la perception agit comme une relance, comme une occasion de processus, et sans doute un processus continu. Même quand nous dormons, nous continuons à muser ${ }^{9}$. Nous en avons l'expérience, mais nous en avons surtout l'expérience quand nous le découvrons, parce que lorsque nous musons, nous ne savons pas que nous musons, nous sommes "en musement", mais nous ne savons pas que nous y sommes, nous ne pensons pas au musement que nous vivons. Par quoi y accédons-nous? Par un drôle de processus: à un moment donné, quelque chose vient faire obstacle à ce flot du musement. Le musement, pour reprendre les catégories de Peirce, de l'être-là de sa priméité, devient objet secondal de l'inscription, étant un continuum troisième.

Le musement, ce n'est pas quelque chose à quoi nous pensons: quand nous sommes dans le musement, et que nous ne sommes que dans le musement, nous ne pouvons avoir une idée de ce 
qu'est ce musement. Quand nous y pensons, d'une certaine façon nous ne sommes plus dans le musement, puisque c'est une pensée consciente cette fois-ci, mais quand nous en avons une pensée consciente, nous savons que nous avons musé. Ce mot devrait d'ailleurs toujours se mettre au passé pour pouvoir le reconstituer: «ah! j'étais en train de penser à quelque chose, ah! oui je pensais à ça, et puis avant cela je pensais à ça», et vous vous retrouvez à faire une remontée dans le musement. C'est l'approche propre du musement par la pensée, il est objectifié: on pourrait parler d' «objet musant» à propos de ce «musé». On a certes une possibilité d'y accéder, mais le musement excède tout ce à quoi l'on peut accéder, parce que nous sentons bien que nous sommes devant un flot, quelque chose de continu. Ce musement-là, je ne peux le connaître que lorsqu'il est au passé, que dans un temps du passé. Je ne peux évidemment le saisir ni dans le présent, puisque j'y suis, ni dans le futur, puisque je ne sais pas ce que sera le musement.

\section{HIÉRARCHIE DES FONCTIONS SCRIBE ET MUSEUR}

Ainsi, le scribe est premier et le museur second. Les descriptions spontanées - c'est-à-dire je muse, puis j'inscris le musement- ne nous préparaient pas à cette idée, mais, depuis Copernic, nous avons pris l'habitude de ne pas nous fier entièrement à l'évidence des descriptions pour saisir l'articulation des phénomènes: à une époque, le soleil tournait autour de la terre. Nous sommes, en cette matière, placés devant cette nécessaire révolution copernicienne.

Sans doute y a-t-il quelque chose qui présente une sorte de contrainte, corporelle, qui fait qu'il y a le langage de l'Autre, du corps, qui est ce musement continu, mais ce qui est premier c'est la discontinuité. Tout commence par une discontinuité. «In principio erat verbum», ou, comme le dit Chouraki dans sa traduction de la Bible «Entête, lui, le logos»: saint Jean était copernicien! Freud précise avec Goethe, «au commencement était l'acte» - c'est sans doute plus clair ainsi. C'est la sortie théâtrale de Totem et Tabou ${ }^{10}$. Bien entendu, Lacan rajoute: l'acte signifiant. «Au commencement était l'acte» est l'introduction d'une discontinuité, une origine, un début. C'est ainsi que nous pouvons entendre que le scribe est premier, c'est lui qui fonde. On peut remarquer qu'il serait tout à fait déplaisant de mettre le museur en premier, puisque nous ne pouvons connaître le musement que par autre chose; le mettre en premier serait rendre compte du connu par l'inconnu. De plus, nous ne pouvons connaître le musement qu'au passé, c'est-àdire quand il est objectivé: on voit bien que pour qu'il soit objet, il faut bien qu'il y ait un signe.

Au commencement était l'acte, parce que l'acte est un acte de langage - le verbe, c'est la parole.

\section{LA FONCTION INTERPRÈTE}

Qu'en est-il maintenant de notre troisième fonction, celle d'interprète? La fonction de base de l'interprète, sa fonction essentielle, est d'expliciter, de dire, de présenter ou de représenter le musement à partir du travail du scribe. Le scribe laisse quelque inscription, et nous savons, par ailleurs, qu'il y a un museur. Je suis amené à parler de museur, de scribe, comme s'il s'agissait de personnes. Je me prévaux en cela de Freud qui, chaque fois qu'il est amené à parler des instances psychiques, les traite comme des personnes ${ }^{11}$. Il montre le moi se bagarrant avec le surmoi, le ça, etc. Finalement, cette méthode sera pratique, elle donne plus de chair à la chose, mais il faudra préciser qu'il s'agit toujours de fonctions. En mathématiques, la fonction sinus s'écrit: sinus $x$. C'est une fonction, c'est insaisissable, et pourtant nous écrivons la fonction sinus $x$ et nous traçons quelque chose, qui n'est pas du tout une fonction, mais qui est l'image de l'ensemble de départ par la fonction, le graphe. Si nous voulons pouvoir saisir une fonction, il faut l'hypostasier, c'est-à-dire lui donner chair, faire comme si c'était quelque chose. Comme ces fonctions sont par excellence humaines, il n'y a aucun mal à les représenter comme des personnes - le savant Cosinus, par exemple!

Nous aurions alors les fonctions suivantes: la fonction scribe représentée par une personne dont le seul travail serait celui d'inscrire; la fonction museur, 
que nous avons illustrée avec Perceval - celui qui muse (mais nous savons bien que nul n'est entièrement consacré à une des fonctions); la fonction interprète se présente de la même manière: comment penser quelqu'un qui ne serait qu'un interprète, qui passerait son temps à dire quel est le musement à partir de l'inscription du scribe? Donc, la fonction de l'interprète, c'est de «duire» (déduire, induire ou abduire) les inscriptions, nous allons dire interpréter, ce n'est pas plus mal. C'est une fonction qui est, bien entendu, essentielle: sans elle, la fonction scribe et la fonction museur seraient vides.

La question de l'interprète est essentielle lors de notre travail avec des personnes en éveil de coma, lors des réunions où l'équipe, avec le blessé, parle du blessé. Selon une décision clinique, épistémologique, éthique, etc., chacun des termes peut être justifié, nous avons décidé que la personne blessée est interprète, c'est-à-dire que nous observons l'interprétation qu'elle fait de nos propres productions. Tous, nous musons: il doit y avoir des connexions "entre» les musements, nous musons tous sur les mêmes choses. Par ailleurs les scribes, manifestement, c'est nous, puisque c'est nous qui parlons. Ainsi la place réservée au blessé est celle d'interprète. C'est une position qui s'est avérée d'un grand usage dans le travail psychanalytique: que sais-je de l'autre? Rien, ou bien peu de choses! Mais ce que je peux faire, c'est dire les choses qui me viennent naturellement, mais en sachant que c'est toujours l'autre qui interprète, celui dont je ne sais pas ce qu'il a dans la tête - nous ne savons jamais ce que nous avons dans la tête les uns des autres. La fonction interprète est une fonction clé, relativement facile à saisir, parce que c'est ce dans quoi nous sommes continuellement, nous nous présentons toujours comme l'interprète des signes.

Quand nous pensons au signe, nous pensons à l'interprétation du signe, toujours, et c'est ce qui rend la sémiotique si délicate. Elle attire toujours l'attention sur le signe inscrit: quand on montre la lune à un imbécile, il regarde le doigt; eh bien, le sémioticien est résolument imbécile, il regarde le doigt! Il est vrai que si le sémioticien ne regardait pas le doigt, il ne verrait pas qu'il indique la lune... C'est tout le travail de la sémiotique en amont de l'interprétation, des interprétants.

Il nous faut maintenant regarder d'un peu plus près la question de l'inscription. Pour cela, nous allons examiner un phénomène mis en évidence à Château Rauzé, où nous travaillons avec des personnes en éveil de coma.

\section{INTRODUCTION À L'ÉCRITURE DES «VÉGÉTATIFS»:} LES TESSÈRES

Un orthophoniste, Van Eeckoute, était venu faire un stage à Château Rauzé pour expliquer aux kinésithérapeutes et aux orthophonistes quelle était sa technique pour faire parler les gens qui ne parlent pas. Il les prend à bras le corps, et, par des poussées sur le diaphragme, fait sortir de l'air, il contrôle la colonne d'air avec la mâchoire et réussit à faire formuler des mots. C'est assez impressionnant. Au sortir de ce stage, une des orthophonistes, impressionnée par cette méthode, a décidé de faire quelque chose de tout à fait étonnant. Elle a mis un crayon dans la main de quelqu'un qui est en phase végétative de l'éveil; se laissant guider par les impulsions du blessé: elle a obtenu une phrase. Elle s'est tout d'abord heurtée au scepticisme général. Puis, des tentatives ont été faites sur d'autres personnes en phase végétative. Or, elles écrivaient toutes. Bien entendu, le contenu est très important: l'une a demandé qu'on lui ramène les boucles d'oreilles qui étaient dans le tiroir de l'armoire de sa chambre, chez elle, ce que personne ne savait; une autre a expliqué ce qui s'était passé la veille et qui n'avait eu comme témoin qu'Edwige Richer, le médecin, qui n'en avait parlé à personne. En quoi cette situation nous concerne-t-elle? Avec les états végétatifs, nous disposons d'un autre moyen de communication, non conscient: la conscience a un sens, mais ces personnes ne sont pas conscientes, ou, du moins, on ne leur prête pas la conscience; ou alors il faudrait alors élargir considérablement la notion de conscience, au point de la vider de sens. 
Lécriture consciente assumée, celle-là a disparu, mais il reste une forme d'écriture basale, corporelle - enfin si nous reprenons ce que nous avons dit plus haut, on voit bien que cela correspond tout à fait à nos hypothèses.

Il faut dire que ce sont des personnes qui ont eu leur accident à une époque où elles savaient écrire. Il y a bien quelque chose qui passe dans l'écriture parce que c'est quelque chose qui vient du corps, non pas par la conscience de ce qu'on est en train de faire, mais justement parce que cela se fabrique, se forge à un tout autre niveau que le pur niveau conscient. Nous arrivons à écrire quand nous arrivons à oublier que nous écrivons, et quand nous oublions que nous écrivons, cela veut dire que l'écriture est devenue intégralement corporelle. Si ces blessés arrivent à écrire, il s'agit là d'une démonstration tout à fait extraordinaire. Cela dit, il a fallu une certaine fraîcheur d'âme pour aborder tous ces problèmes. La «science» n'y suffit pas, la poésie y est bien plus essentielle.

Pour saisir le caractère corporel (au sens du leib, toujours) de l'écriture, que l'on songe au pianiste qui travaille un morceau un peu difficile, se trompe toujours sur un certain passage, se repose une nuit et le lendemain joue le morceau sans faute. C'est un phénomène que nous connaissons bien, mais c'est tout sauf automatique. Nous avons un terme qui le décrit: c'est un acte tessérisé (ces tessères corporelles que nous avons déjà évoquées).

\section{LES TESSÈRES}

Les tessères? Maintenant il n'y a plus de linotypistes. Le linotypiste était face à un clavier, dans les imprimeries, et en tapant sur une touche, il faisait couler du plomb qui venait fabriquer une lettre; il composait la page avec du plomb, grâce à sa linotype. La tessère est l'analogue de ces morceaux de plomb, c'est quelque chose de matériel, mais qui porte une inscription, c'est le porte-type, comme le dit Edwige Richer ${ }^{12}$. Nous avons supposé depuis longtemps que pour qu'il puisse y avoir langage de quelque manière que ce soit, il était important d'envisager que le corps
- mais ce n'est pas quelque chose qui est rassemblé comme le sont les morceaux de plomb, c'est beaucoup plus diffus - comportait toutes les tessères possibles. Nous avons "en corps" les tessères de tout ce que nous pouvons penser et dire. La tessère fondamentale de l'ensemble, c'est le corps: on pourrait dire que le corps "comme tel» n'est autre que la feuille d'assertion, le lieu de l'inscription, qui, «blanche», représente l'Univers du discours.

Le musement est corporel, le musement s'appuie sur des tessères corporelles. C'est le musement du corps, le corps qui pense à notre place. Notons que sans cette hypothèse, on ne voit pas comment les personnes en phase végétative pourraient écrire. Il y a bien quelque chose qui est corporel, qui a la matérialité du corps, mais qui est porteur d'inscription.

Nous avons l'habitude de dissocier ce qui est du registre de la pensée et ce qui est de celui du corps. En fait, cette dissociation, nous l'avons héritée de temps immémoriaux, mais elle n'est absolument pas pertinente. Elle nous conduit à des contradictions tout à fait extraordinaires, c'est-à-dire que si nous avons quelque chose comme la pensée qui est séparée du corps, on ne voit pas du tout comment, à un moment donné, quoi que ce soit de la pensée peut interagir avec le corps. Ce qui rend ces choses difficiles à penser, ce sont nos habitudes. Mais si nous décidons de considérer un "corpensée», alors nous accédons à l'idée de tessère, qui comporte à la fois quelque chose du registre du corps, mais aussi quelque chose du registre de la pensée, une émergence de pensée possible.

\section{TYPE/TESSÈRE}

Depuis près d'un siècle, les logiciens ont pris l'habitude de faire une distinction, sans doute très importante dans notre champ, entre "type» et "token", que nous traduisons habituellement par «type» et «tessère». Il est aisé de saisir la signification de cette différence dans ce métier auquel nous venons de faire allusion, la typographie, qui a, sous sa forme classique, quasiment disparu. Quand les linotypistes 
fabriquaient les plombs dans lesquels ils fondaient les lettres, les mots, les espaces, les signes de ponctuation, quand ils faisaient ce travail de typographes, ils fabriquaient en somme des matières, les tessères, à travers lesquelles nous pouvions lire de belles histoires... c'est-à-dire les lettres, les mots, etc. D'ailleurs les typographes avaient très bien compris qu'ils faisaient quelque chose de tout à fait essentiel pour l'humain, puisqu'ils faisaient nommément des caractères d'écriture. (Il faut savoir que, dans l'argot des typographes, il y avait deux types d'ouvriers: ceux qui travaillaient à la tâche et ceux qui travaillaient à la journée. Un ouvrier qui travaillait à la journée s'appelait une conscience. Il y a quand même là des affinités entre la typographie et la dimension de l'humain, de l'être parlant sans doute.)

"Tessère" vient du latin tessera qui, lui-même, vient du grec et signifie le cube ou le carré. En fait, les tessères sont les jetons de présence. Quand nous entrons dans une boîte de nuit par exemple, on nous tessérise le corps, on nous tamponne la main, on nous donne une contremarque. Dans les jeux de cirque, on donnait des morceaux d'argile aux personnes qui avaient payé leur billet. Bref, quand nous prenons un billet, une tessère, cela signifie que nous sommes inscrits. Ce n'est pas l'inscription elle-même qui, elle, est beaucoup plus complexe, c'est le témoignage matériel d'un contrat - voilà l'idée de tessère - et nous pouvons penser le corps de cette façon.

Les tessères font en sorte que l'organisation interne du corps, du leib, est quelque chose qui a à voir avec l'organisation des signes, puisque, d'une certaine façon les tessères corporelles ont leur propre logique, corporelle, matérielle. Regardons le grand pas, dans l'histoire de l'écriture, qu'a représenté l'invention du blanc, ce qu'on appelle une espace. Une espace est quelque chose qui est inséré entre des lettres typographiées de manière à séparer les mots. Les Grecs ne le faisaient pas, c'est une invention tout à fait extraordinaire parce qu'elle a permis l'organisation des mots, qui était implicite dans le discours et qui devenait explicite dans l'écriture - logique interne de la typographie. Prenons cette logique de la typographie, de l'écriture, de l'écrit et essayons de penser ce que peut être cette même logique pour l'écrit que constitue le penser corporel.

Bien entendu se pose la question de la conscience qui, pour nous, est toujours prééminente - c'est ce qui nous permet de savoir qu'on sait. Savoir et savoir qu'on sait, ce n'est pas du tout la même chose.

Rappelons à ce propos comment se définit le possible, au moins le possible dit subjectif: le possible est ce que je ne sais pas ne pas être. Par exemple, dire «il est possible que demain il pleuve» signifie: je ne sais pas que demain il ne pleuvra pas. La possibilité de tel événement n'est autre que le fait de ne pas savoir que cet événement ne se produira pas. La possibilité suit une logique négative. Cette définition du possible remonte au Moyen Âge, mais ne couvre sans doute pas tout le champ du possible. Si je dis, par exemple, «il est possible que je lève le bras», cela ne veut pas dire: je ne sais pas que je ne le lèverai pas. C'est une possibilité dite objective. Possibilités subjective et objective sont assez bien exprimées par les verbes anglais may et can. May I tell you something? Je ne sais si quelque chose s'oppose à ce que je le dise. I can tell you something. Rien ne s'oppose à ce que je dise. On voit là la négation opérer dans les deux genres de possibilités.

Reprenons le scribe. On voit bien qu'il est important de pouvoir considérer, dans cette fonction scribe, quelque chose de particulier qui sera la fonction d'écriture, au sens d'une production matérielle. Le scribe inscrit, mais pour cela il faut bien quelque matérialité, il faut quelques tessères, c'est sa fonction scriptive, c'est-à-dire sa fonction de production matérielle. La fonction scriptive peut être parfaitement distinguée - mais pas séparée - des autres, elle peut être mise en acte par quelqu'un d'autre.

Si nous reprenons la personne à l'état végétatif en train d'écrire, matériellement l'essentiel est fait, la scription est faite à deux, avec la personne qui accompagne la main, qui permet d'ajuster la pression sur la feuille de papier, mais en obéissant à des ébauches de gestes. Comment pouvons-nous interpréter les ébauches de gestes? Comme des ébauches d'inscription. La personne nous donne une 
sorte de direction qui a quelque chose à voir avec l'inscription. On voit bien que, analytiquement, tout cela est lié.

\section{LA TRICHOTOMIE DE LA FONCTION SCRIBE}

Depuis longtemps, nous avons fait une distinction à l'intérieur de la fonction scribe. Voici les trois fonctions liées à la fonction scribe. Nous avons le scribe proprement dit, qui est la fonction d'écriture ou de scription (fonction scriptive); une deuxième fonction que nous appelons la fonction pythique; et une troisième fonction que nous appelons la fonction mantique.

Je préciserai ces trois fonctions à partir de l'histoire de la pythie de Delphes ${ }^{13}$. La pythie de Delphes était une prêtresse d'Apollon, qui avait comme mission, en dehors de celle de fêter Apollon, de rendre des oracles; la pythie de Delphes était très célèbre dans l'Antiquité parce qu'elle avait rendu des oracles fameux (en particulier celui sur Crésus). Elle avait son jour de consultation.

Pour recevoir un oracle, que fallait-il faire? L'oraculant (celui qui postule à recevoir l'oracle) était reçu dans une pièce spéciale qui s'appelait l'Adyton, par ceux qu'on appelait les herméneutes. Ces herméneutes, je les ai baptisés les manticiens. La mantique est l'art du devinement, le manticien est celui qui devine. Donc, l'oraculant arrivait chez le manticien, à qui il disait ce qu'il voulait savoir: la fonction du manticien était alors de lui dire comment il fallait poser la question à la pythie. Le lendemain arrivait le grand moment de la rencontre avec la pythie, assise sur un trépied au-dessus de la faille. L'oraculant posait sa question et, après un moment de recueillement, elle poussait des cris. Bien entendu, l'oraculant ne comprenait rien aux cris, il lui fallait un traducteur. Il retournait donc à l'Adyton, où le manticien traduisait ce qu'avait dit la pythie, généralement sous la forme d'une énigme, un petit poème, deux, trois ou quatre lignes, parfois une ligne, peut-être selon la durée des cris de la pythie.

L'interprète, ce n'est pas le manticien mais l'oraculant, suivant la conduite à laquelle donnait lieu la «traduction» de l'énigme. Lorsque la pythie de Delphes lance à Crésus: "Quand un mulet sera roi des Mèdes, ne rougis pas de fuir, ô Lydien, le long du fleuve Hermus», Crésus crut son royaume indestructible! Or Cyrus, son vainqueur, était bel est bien un «mulet», fils d'une jument, une princesse mède, et d'un âne, un Perse de condition modeste.

Nous voyons ainsi que le manticien intervient dans l'inscription, il n'intervient pas dans l'interprétation, fondamentalement, de l'oracle. La production de l'oracle est le fait d'une triple coopération, celle de l'inspiration divine de la pythie, celle des cris et de l'écrit du manticien-scripteur et enfin la force de devinement du manticien.

La fonction scribe est donc composée de trois fonctions: fonction scriptive ou tessérique; fonction pythique, qui est proche du musement; fonction mantique, qui est celle du devinement. Les trois fonctions collaborent à la production de l'inscription, c'est-à-dire de l'interprétation possible. Dans l'exemple de Château Rauzé, la fonction mantique est dans le geste de l'orthophoniste, qui devine vers où la pythie l'amène, elle soutient le poignet, elle assure la pression sur la feuille. Certes cela implique la fonction scriptive, mais ce n'est pas tout. Elle va obéir aux impulsions pythiques de musement du végétatif. Elle devine, ce qui est le point important, et cette fonction mantique est celle que nous pensons avoir auprès des personnes en éveil de coma. Quand nous travaillons avec elles, nous devinons les choses et, les ayant devinées, nous les laissons interpréter par leur réaction (un sourire, un grognement, parfois même une parole, etc.).

On devine, on assure la fonction scribe, par la fonction scriptive et mantique, laissant la fonction pythique au blessé; puis nous le mettons en position d'interpréter ses propres paroles. On voit que dans les éveils de coma, il y a comme une dissociation; la personne qui produit quelque chose ne sait pas ce qu'elle a produit: la reprise de conscience est justement le moment où ces choses vont pouvoir aller, la fin de l'extrême dissociation.

Cette «théorie» a comme seul mérite avoué celui de nous permettre d'observer ces choses, d'en rendre 
compte, de ne pas être en contradiction avec ce qu'on observe. Elle a comme seule attente de nous permettre d'observer des choses nouvelles.

La mère pythique, elle, va occuper les trois fonctions. Elle va dire, par exemple, au troisième mois de l'enfant: «il a souri». En fixant le sourire de l'enfant, elle est dans la fonction scriptive. Comme cette fonction ne peut pas être détachée des autres, c'est la signification du sourire qui est impliquée, ce n'est pas le sourire, pas le rictus. Dans un deuxième temps, la fonction pythique, c'est l'être de la mère avec l'enfant. Il y a là quelque chose de pythique, un mélange, une confusion presque corporelle. L'enfant nourri au sein par exemple, bien porté, c'est d'une confusion totale. On ne sait plus qui est qui, on sait tout ce qui se passe chez l'enfant, parce que le corps n'est pas totalement différencié. Dans un troisième temps, la fonction mantique, c'est celle que j'appelle la mamanticienne, celle qui devine et qui, devinant, inscrit. Parce qu'elle devine, elle inscrit - dans le sourire, elle devine quelque chose.

On peut alors considérer que l'absence de l'une de ces trois fonctions qui concourent à la fonction scribe empêche l'inscription. Cependant, comme il s'agit des fonctions, et si nous songeons aux personnes, elles peuvent donner lieu à des substitutions. Dans ce qu'on peut appeler l'invention de l'enfant, l'«invention» consiste d'abord dans ce phénomène de l'inscription.

Précisons encore ces fonctions. Tout ce que la pythie produisait était sous l'inspiration d'Apollon, puisqu'elle était en contact direct avec lui. Certes la demande de l'oraculant fournissait l'occasion, mais le point de base était qu'elle recevait l'inspiration d'Apollon: elle était donc la manticienne et la scriptrice de celui qui était en position pythique à ce moment-là, c'est-à-dire Apollon. C'est un processus qui n'a pas de fin, ni d'un côté ni de l'autre. Une sorte d'objet fractal.

Nous décidons d'être à un certain niveau de lecture, comme avec les traumatisés crâniens, pour qui nous pouvons très bien dire qu'ils sont interprètes, dans la situation qui est à saisir. Quand nous sommes dans l'analyse de la fonction scribe, nous voyons cependant que le blessé est plutôt du côté pythique et nous du côté manticien: scripteur et devineur. Nous voyons bien que les places sont différentes suivant les endroits où l'on se situe et la finesse des fonctions qui permettent d'y accéder. Parfois, c'est le blessé qui, par son interprétation, peut devenir le manticien pour nous. Il est évident que lorsqu'un blessé qui est à l'état végétatif se met à pleurer, il touche en nous quelque chose, il a deviné un sentiment d'émotion qui jusquelà n'avait pu s'extraire.

Ce modèle est à prendre comme quelque chose qui est à articuler chaque fois dans différentes positions, et chaque fois il faut voir ce qu'il peut nous permettre de produire. Qu'est-ce que le fait de placer le blessé en position d'interprète peut nous permettre de produire, sinon de le respecter? Je ne sais pas ce qu'il pense et je persiste à ne pas savoir ce qu'il pense. Donc c'est à lui de nous dire si ce que nous sommes en train de proférer est interprétable ou pas. Par ailleurs, nous ne savons pas ce qu'il interprète de nos inscriptions, il nous dit simplement que dans tout ce qu'on a raconté, il y a quelque chose qui a été touché. On a dû toucher quelque chose, on a donc inscrit quelque chose qui lui a permis d'interpréter.

La position inverse, celle qui consisterait à interpréter le blessé, est une position terrible. On le considère et l'on dit ce qu'il pense. Il y a un risque, on peut le mener à des horreurs. Parfois, nous n'échappons pas au fait de ne pas être toujours dans cette position, parce que sinon, cela fait longtemps qu'ils auraient écrit; il a fallu une orthophoniste poète, avec la fraîcheur nécessaire pour faire ce qu'on appelle en analyse une interprétation. Une interprétation en psychanalyse est mantique, elle n'est pas du côté de l'interprète, mais du côté du manticien. Le patient repart avec son oracle, et c'est lui qui interprète, comme nous le savons, puisque l'interprétation se fait toujours après coup. Quand nous interprétons, nous ne savons pas que nous sommes en train de le faire. Nous pouvons dire que le manticien est en règle avec la pythie, il ne sait pas ce qu'il a interprété. 
Les rapports entre le scribe et le museur doivent être des rapports de familiarité, parce que, sinon, nous ne voyons pas très bien comment nous pourrions inscrire le musement. La plupart du temps, le scribe et le museur sont une seule et même personne; ces fonctions, nous pouvons les distinguer, mais pas les séparer; il faut une certaine familiarité. Moins de familiarité est demandée à l'interprète, puisque le savoir - un certain savoir sur l'inscription et ses règles peut suffire. Nous pouvons interpréter les hiéroglyphes, mais il n'est pas nécessaire pour autant d'être très familier avec la civilisation égyptienne, même si la familiarité pourra garantir une interprétation plus complète (il n'empêche que nous pouvons donner une interprétation tout à fait intéressante). Il a pourtant fallu à Champollion une réelle familiarité pour pouvoir les deviner. Le rapport scribe-museur et le rapport scribe-interprète ne nécessitent pas le même genre de familiarité. Autant le museur et le scribe doivent être familiers l'un avec l'autre, autant nous pouvons imaginer une distance plus importante entre le scribe et l'interprète. Cela correspond à la distinction de William James entre «savoir sur» (knowledge about), savoir d'interprète, et «savoir de familiarité» (knowledge by acquaintance), savoir de scribe.

On peut se poser la question suivante: est-ce que la pensée ne vient pas aux enfants parce que la mère tessérise le corps? (Autrement dit, la mère, la mère type - Lacan parle de la mère symbolique.) Parce qu'à ce moment-là, tout ce dont elle est porteuse en termes de types, elle en forge les tessères dans ou avec ou par le corps de l'enfant. Elle le tient d'une certaine façon dans les postures, elle l'accompagne dans le mouvement de son corps, elle l'initie à ses tessères. Tessères que l'enfant va dans un premier temps vivre dans son corps avant de pouvoir, d'une façon ou d'une autre, les lire. Et qu'est-ce que l'enfant qui lit les tessères de son corps? Eh bien, c'est le museur, le «Mind-reader» dont parle Peirce. C'est un lecteur, un lecteur infatigable des tessères du corps. Il lit dans toutes les langues du monde, il ne se fixe pas à une langue, comme la plupart d'entre nous.
La tessérisation du corps est une opération qui se fait sous la houlette de la mère, de la mère symbolique, qui introduit l'enfant aux types. L'enfant, lui, muse sur, avec ou par son propre corps.

\section{LA FEUILLE D'ASSERTION}

Qu'en est-il maintenant de ce que, plus haut, nous avons appelé la feuille d'assertion? C'est ce qu'il nous faut bien supposer pour recevoir l'inscription dans toutes ses dimensions. Il faut écrire des tessères, ou bien parler. Cette feuille d'assertion, il me semble qu'on peut en trouver l'ébauche dans ce doublet de Winnicott: l'espace transitionnel et l'espace potentiel. La feuille d'assertion serait du côté de l'espace transitionnel. C'est, en somme, la feuille de transactions avec l'autre. L'espace potentiel, lui, serait vraiment cet espace que Peirce appelle le lecteur d'esprit... le museur. Mais il faut voir que l'esprit (mind en anglais) a des connotations infiniment plus concrètes que l'esprit «français».

Du point de vue du médecin, il doit s'assurer qu'il y a une feuille d'assertion pour qu'un scribe puisse inscrire les choses. Un musement continu qui ne s'inscrit jamais, c'est l'équivalent d'un état végétatif. Il faut donc pouvoir inscrire, mais pour pouvoir inscrire il faut une feuille d'assertion. Cette feuille d'assertion, c'est l'espace transitionnel. C'est une opération de l'Autre, du corps-leib, et, fondamentalement, comme opération de l'Autre, elle est en lien avec le sujet. Mais le corps est bien plus complexe que la complexité anatomique: dans l'autisme, par exemple, le corps va même jusqu'au coin de la pièce, il peut être ailleurs, il est peut-être situé à quelques kilomètres de là, dans un endroit qu'on ignore.

Si la feuille d'assertion est un corps, alors elle peut être un corps à plusieurs. Mais le corps à plusieurs, c'est encore un corps, et c'est ce qui est important. Elle peut être le corps d'une équipe, elle peut être un corps que, parfois, il faut découvrir, car le seul fait de savoir, de connaittre la feuille d'assertion, peut être un renseignement magnifique sur l'état d'une personne. Mais parfois la feuille d'assertion ne permet pas d'asserter grand chose. On peut dire que quand elle 
est trop dispersée, elle ne peut plus inscrire. S'il y a trop de trous dans une feuille d'assertion - nous pouvons écrire tous les discours dans les trous -, eh bien c'est raté.

Cette idée que les choses puissent s'inscrire rend des services pour différentes équipes, mais aussi pour le psychanalyste. En quoi consiste le travail d'un psychanalyste? Que les choses puissent s'inscrire! L'interprétation, il ne sait pas la faire, c'est au blessé de la produire. Le psychanalyste se borne à permettre, à fournir un dispositif pour que ça puisse s'inscrire. $\grave{A}$ l'autre d'interpréter.

La question est donc toujours la même: est-ce que c'est inscrit? Est-ce qu'on a vraiment un dispositif qui permet de s'inscrire? Du seul fait d'avoir disposé une feuille d'assertion, immédiatement tout change, parce que, précisément, il se met à y avoir un libre jeu de ce corps.

Quel est le statut de l'écriture des blessés en phase végétative de l'éveil de coma? Ces sortes de mouvements, ces mélodies kinésiques, renvoient aux tessères corporelles dont je parlais. On aurait là quelque chose qui s'avère être une écriture qui serait plus archaïque que la parole. Bien entendu, dans le déroulement temporel, il y a peu de doute, l'écriture arrive après la parole. Et pourtant, il faudrait arriver à cette conclusion: l'écriture est plus archaïque, au sens de plus intime, que la parole. Au lieu de penser l'écriture comme un moyen de communication, on pourrait la considérer comme un journal intime, celui du sujet dans son dialogue avec l'Autre. L'écriture est intime, quoiqu'un peu «extime» tout de même. Et l'on se rend compte qu'il y a quelque chose, en fait, d'extrêmement profond, qui touche l'essence même de l'intimité du sujet, qui est, peut-être, du registre scriptural.

On parle beaucoup des «enveloppes». Or qu'y a-t-il dans les enveloppes? On le sait bien, des écrits. Surtout quand les enveloppes sont timbrées! Car, quand les enveloppes ne sont pas timbrées, elles peuvent être vides. Quelle est la fonction de ces enveloppes? C'est de receler des écrits cachés. Je n'aime pas trop le dualisme, même si parfois j'y tombe comme tout le monde, en particulier celui des contenants et des contenus. Je ne crois pas qu'il y ait des contenants et des contenus. Même le «moi-peau». Je préfère les membranes à la peau. C'est quand même plus astucieux, les membranes, parce que ce sont des lieux d'échanges beaucoup plus nets... Enfin, peut-être ne faudrait-il pas penser les enveloppes en termes de contenant, mais les penser comme là où sont déposés les écrits secrets. C'est-à-dire les premières écritures fondamentales, c'est-à-dire, au bout du compte, les tessères qu'on pourrait appeler des tessères primordiales, qui sont peut-être ce que Jean Oury développe en parlant du narcissisme originaire.

\section{CONCLUSION}

Ce que nous venons de présenter ici n'est qu'une introduction. Nous venons simplement de poser les premiers repères fondamentaux de la clinique, d'une clinique poïétique, à savoir un dispositif permettant de placer les rapports du ou des «thérapeutes» et du «patient». Pour résumer brièvement, le patient est constamment mis en position d'interprète alors que le thérapeute est en position de scribe - l'objet étant alors produit par la discontinuité introduite par le scribe dans le musement «du» patient. Il s'agit d'un principe fondamentalement logique, s'appuyant sur une éthique. Cette éthique pourrait être formulée ainsi: «laisser le patient être l'interprète de ses propres objets». Mais cette éthique a pour fondement une esthétique. Nous avons tenté d'en donner quelques aperçus. Le surgissement de la forme nous amène à la saisir non sous les espèces d'un objet, l'objet-forme, mais bien plutôt comme un rythme, en laissant le terme rythme dégagé par rapport à l'espace et au temps. Cette forme-rythme, que nous avons appelé ici "forme émergente», ne saurait être conçue sans une secondéité, qui est celle du musement, et une tiercéité, qui est son interprétabilité: elle est en cela au fondement même du «tonal» dans son rapport au musement. Les conditions du laisser-être de ces formes (priméité), dont le sujet-museur se fait comme le lieu (secondéité) parce qu'il en est l'effet (tiercéité), sont le principe de cette esthétique. 
D'autres développements sont réalisés sur la question du sujet, de la pulsion, etc. Dans le cadre de cet article, nous n'avons pu aller plus loin: le lecteur est déjà largement mis à contribution, plus qu'il ne serait désirable, pour incorporer les quelques abstractions que ces pages contiennent.

\section{NOTES}

1. Il habitait auprès du rempart; il s'alita, le premier jour il eut une fièvre aiguë avec sueur; nuit pénible. Le deuxième jour, aggravation générale; le soir, évacuations favorables après lavement; nuit tranquille. Le troisième jour au matin et jusqu'au milieu de la journée, il parut sans fièvre; mais venu le soir, fièvre aiguë avec sueur, soif, la langue commence à devenir sèche, il rendit des urines noires; nuit pénible, il ne reposa point, confusion mentale sur toutes choses. Le quatrième jour, exacerbations générales, urines noires; nuit plus supportable, urines de meilleure couleur. Le cinquième jour, vers le milieu de la journée, léger écoulement du nez d'un sang non mélangé; urines variées avec des suspensions flottantes arrondies, semblables au sperme, dispersées; pas de dépôt. Après un suppositoire, peu d'excréments avec des vents. Nuit pénible; courts sommeils, discours, divagations; extrémités froides partout, impossibles à réchauffer; il rendit des urines noires; assoupissements brefs à l'approche du jour; sans voix; sueurs froides; extrémités livides. Il mourut vers le milieu du sixième jour. Il eut jusqu'à la fin un souffle grand et rare, comme s'il cherchait à le rappeler. La rate s'éleva en formant une enflure arrondie; sueurs froides jusqu'à la fin. Les paroxysmes aux jours pairs.

2. Hippocrate, $1986: 15$ sq.

3. Cf. notre thèse de 1986 éditée en 2000 sous le titre Des fondements sémiotiques de la psychanalyse.

4. Pour la logique de ces concepts, voir particulièrement Psychanalyse, logique, éveil de coma: le musement du scribe.

5. Et tout d'abord à Peirce lui-même dans les Collected Papers (1.521-

544).

6. C'est ainsi que nous traduisons le terme "representamen " de Peirce (cf. M. Balat, 2000a).

7. R.A. Spitz, 1968: 68sq.

8. Les Romans de Chrétien de Troyes, t.V, Paris, Honoré Champion, 1990, p. 131sq.

9. Nous sommes en plein débats, depuis des années, avec mes amis du trauma crânien, parce que je soutiens que les gens qui sont dans le coma musent, eux soutiennent que non. Ce n'est pas une question très importante, même si on peut leur demander ce que voudrait dire «reprendre" le musement.

10. S. Freud, 1998: 189sq

11. S. Freud, 1995: 160sq.

12. Cf. M. Balat, 1998.

13. Cf. l'article "Oracle» de M. Delcourt dans l'Encyclopædia Universalis.

\section{RÉFÉRENCES BIBLIOGRAPHIQUES}

BALAT, M. [2000a]: Des fondements sémiotiques de la psychanalyse: Peirce et Freud après Lacan, Paris, L'Harmattan;

[2000b]: Psychanalyse, logique, éveil de coma: le musement du scribe, Paris, L'Harmattan, 2000;

(sous la dir. de) [1998] : Autisme et éveil de coma, Nîmes, ThéétèteLe Champ social.

BENVENISTE, É. [1969]: Vocabulaire des institutions indo-européennes, Paris, Minuit.

FREUD, S. [1998] : Euvres complètes, t. XI, Paris, P.U.F.; [1995] : Euvres complètes, t. XIX, Paris, P.U.F.

Hippocrate [1986] : La Consultation, Paris, Hermann. Peirce, C. S. [1931-1935]: Collected Papers, vol. 1-6 (sous la dir. de C. Hartshorne et P. Weiss), Cambridge (Mass.), Harvard University Press; [1958]: Collected Papers, vol. 7-8 (sous la dir. d'A.W. Burks),

Cambridge (Mass.), Harvard University Press.

SPITZ, R.A. [1968] : De la naissance à la parole, Paris, P.U.F.

Winnicot, D. W. [1969]: De la pédiatrie à la psychanalyse, Paris, Payot. 